\section{Effects of neuroleptic medication}

\section{DeAR SirS}

Chapman \& Mulvihill's paper on perceptions of the clinical and psychosocial effects of neuroleptic medication by patients with schizophrenia (Psychiatric Bulletin, 1990, 14, 331-332) produced two surprising results. Firstly, the reported response rate was $72 \%$ from a postal questionnaire, and secondly, the overwhelming majority of patients responded favourably to questions about medication. These results were unexpected from a group of patients whose internal and external worlds are often chaotic, and at odds with our clinical experience of having repeatedly to persuade patients of the benefits of remaining on medication. We therefore set out to determine if those patients seen most often by hospital doctors are less contented with treatment.

Postal questionnaires asking about perceived benefits and side effects of neuroleptic medication were sent to members of the Oxford branch of the National Schizophrenia Fellowship (NSF), a similar organisation to the original study. For comparison, questionnaires were given to patients receiving depot neuroleptics at hospital run facilities, and at general practice health centres served by the same psychiatric team. Patients receiving depot medication were chosen as they were easily identifiable, and were thought more likely both to have seen a psychiatrist and suffer from schizophrenia.

Of 58 questionnaires sent to NSF members, 14 were completed and returned $(24 \%)$, ten from subjects receiving depot medication. Ten out of 16 hospital patients $(62 \%)$ and nine out of 17 health centre patients $(59 \%)$ agreed to complete the questionnaires. Those who refused to participate were known to be mentally unwell or refusing medication at the time. The groups were similar with respect to past history of illness.

Of those patients receiving depot medication, 24 out of $29(83 \%)$ thought medication was quite or very important in aiding recovery from illness, and a similar proportion thought it important in helping them to remain well. Seventeen out of 29 patients $(59 \%)$ reported that side effects had interfered with their life "quite a lot" or "very much". Weighing up the benefits and side effects of treatment, 17 out of 29 $(58 \%)$ were "quite" or "very" satisfied with their medication.

The response rate of $24 \%$ from NSF members in this study is closer to that which we expected. This suggests the previous study population was biased and the replies were from an atypical group of patients. The response rate from the other two groups was better but patients were approached personally. Information available about non-responders suggests that these patients were less compliant and more dissatisfied with their medication. With a low response rate, it is difficult to derive firm conclusions from the findings. It is clear, however, that many patients recognise benefits from neuroleptics despite suffering concomitant side effects. They appreciate being asked about side effects and their reports being taken seriously. They also appreciate trials of alternative medication which may lessen their drug induced handicap.

\section{Research Institute for the \\ Care of the Elderly \\ St Martin's Hospital \\ Bath BA2 5RP}

Whitchurch Hospital

Cardiff

ANDREW BARKER

The Elms Clinic

Horton General Hospital

Banbury

\section{"Cannabis psychosis"}

\section{DeAR SIRS}

I am the only consultant psychiatrist for the Tairawhiti District on the East Coast of the North Island of New Zealand, which has a population of 48,000 , and covers an area almost as large as Wales. The coastal area to the north of Gisborne has long been notorious for the growing of cannabis, offering for the grower the best sunshine in New Zealand and the lowest police presence. Over the last year, there have been an average of two acutely psychotic patients transferred per month from the East Coast to the Gisborne Hospital for treatment. Because of the high incidence of cannabis use in that area among the unemployed (recent estimate equals $90 \%$ unemployment), we routinely conduct drug screens on urine specimens. Of the last 15 such admissions from the East Coast, 10 have proved to be positive for urinary cannabinoids. These patients tend to get well quickly with a minimum of treatment.

We have, too, had the experience of such patients being discharged, free of psychotic symptoms, only to return with a florid relapse, and biochemical evidence of further consumption of cannabis. This type of evidence is difficult to refute, and my feeling is that the nosological status of "cannabis psychosis" will not remain unclear for many more years.

It is because there is no specific symptom cluster associated with cannabis use (i.e. the symptoms can mimic other psychotic states), that the entity of cannabis psychosis is eschewed in the literature. The thinking is confused. Because the symptoms occur in other states, how can it be argued that the entity does 